\title{
Comparative Analysis of Three Dimensional Channel Models in Molecular Communication via Diffusion
}

\author{
Anmol Preet Kaur ${ }^{1}$, Dr. Deep Kamal Kaur Randhawa ${ }^{2}$ \\ Student, ECE Department, Guru Nanak Dev University, RC Jalandhar, Punjab, India ${ }^{1}$ \\ Senior Assistant Professor, ECE Department, Guru Nanak Dev University, RC Jalandhar, Punjab, India ${ }^{2}$
}

\begin{abstract}
The movement of messenger molecules in molecular communication via diffusion (MCvD) follows Gaussian distribution function. Due to the nature of random movement of information molecules (or messenger molecules), one of the key challenges is to reduce inter- symbol interference (ISI) that results from the response of diffusion process occurs in Gaussian distribution channel. In this paper, we propose and evaluate a new Poisson distribution channel model for molecular communication which is a novel solution to improve the system reliability and to reduce the impact of ISI. In this approach, now the randomly propagating messenger molecules follow Poisson distribution function. This paper analyzes and compares these two approximations of the binomial distribution of propagating molecules in 3-D MCvD system. We investigate either Poisson or Gaussian channel model is better in terms of ISI, Symbol Error Rate (SER), and communication delay via numerical simulations.
\end{abstract}

Keywords: Molecular Communication via Diffusion (MCvD), Ligand-Receptor Binding Process (LRBP), binomial, Gaussian, Poisson, Additive White Gaussian Noise (AWGN).

\section{INTRODUCTION}

Nano-technology is the manipulation and development of devices within the nano- meter scale and these devices are called nano- machines. A nano- machine is a tiny machine on the scale of 1 to 100 nano- meters that is capable of functioning simple tasks such as data storing, computing, and sensing [1]. At nano- scale, the physical, electrical, chemical, optical, and mechanical properties of the material changes significantly. Cooperation and communication among nano- machines expand its capability to carry out complex tasks such as health monitoring, environment monitoring and tissue engineering [2].

It is difficult to apply conventional communication techniques, namely electro- magnetic, acoustic and magnetic communication within the nano- scale devices. Instead different alternative methods have been proposed for communicating nano- machines. One of these methods is Molecular Communication (MC) and it is a new bio-inspired paradigm where chemical signals or molecules are used to transfer information between a pair of nano- machines. In MC molecules are used to encode outgoing information, transmit these molecules in propagation medium and receive these molecules to decode the transmitted information. MC system can be classified into different categories such as ion signaling, molecular communication via diffusion [3].

Among the MC system, a low complexity and energy efficient method for transferring information is Molecular Communication via Diffusion (MCvD). A MCvD system consists of a transmitter nano- machine (TN), a receiver nano- machine (RN) and a molecular propagation medium between them. The TN stays in the fluid medium and they release modulated molecules or information molecules. The information molecules are propagated by mean of the diffusion process through the medium and eventually the molecules are received by the RN [4]. The RN computes the received concentration of the molecules and then decodes the information symbol sent by the TN. In MCvD system, single type of information carrying molecules is used and the information is encoded in the concentration or the amplitude of the transmission rate of information carrying molecules. These information molecules propagate through the environment, following diffusion process, with some portion of molecules hitting at the receiver surface [5].

The chemical receptors placed on the receiver's surface captures the incoming information molecule by means of a chemical reaction process called Ligand-Receptor Binding Process (LRBP) [6]. Each chemical reaction produces an infinitesimally very small electro- chemical signal. Once the propagating information molecules colliding with the chemical receptor, it is assumed that receptor removes the molecule from the environment which means each molecule contribute to the signal only once [5].The MCvD system composed of four main processes as illustrated in figure 1: 
A. Encoding

Encoding is the phase in which TN translates the information into information molecules or messenger molecules so that a RNs can recognize the encoded pattern. Depending upon the properties of molecules (i.e., structure, concentration, sequence, type of molecules, and composing) information may be encoded in multiple forms.

\section{B. Sending}

Sender nano-machine emits information molecules into the aqueous environment. The size of a sender nano-machine contains a limited amount of energy and molecules. By using multiple sender nano-machines may emit same information molecules results in strong signal in the medium.

\section{Propagating}

The information molecules propagate from transmitter nano-machine through the channel to receiver nano-machine.

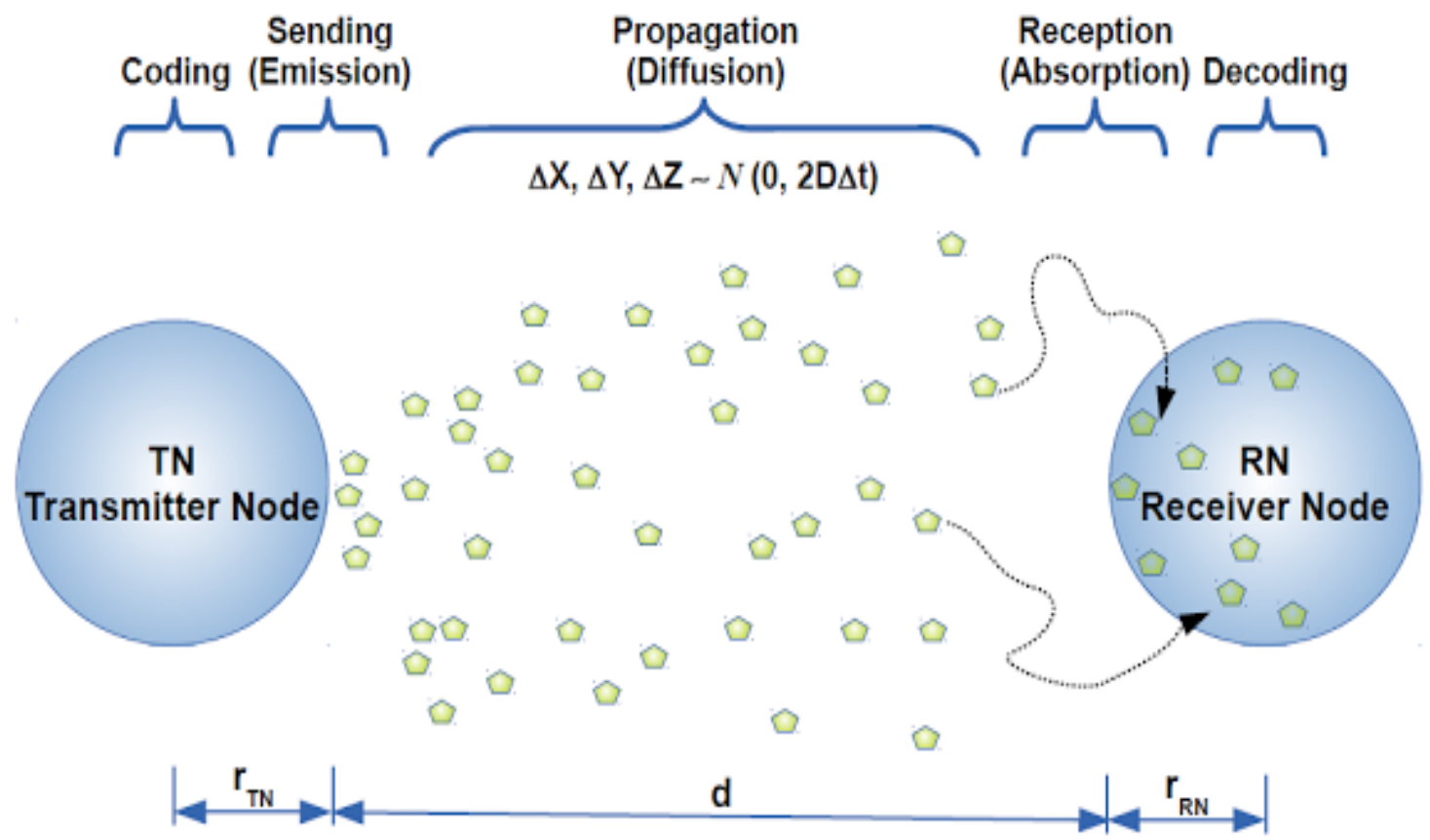

Fig. 1 Three-Dimensional system model in Molecular Communication via Diffusion system

\section{Receiving}

It is the process of capturing information molecules that were diffuse in the environment from transmitting nanomachine. The RN senses the messenger molecules with its chemical receptors located on its surface and then detects the transmitted symbol [7]. In the MCvD, some messenger molecules (MMs) take more time during the journey toward the RN as a consequence of Brownian motion. These molecules are called stray molecules. These stray molecules come into the following symbol transmissions, causing ISI.

The remainder of the paper is organized as follows. Literature survey is presented in section and section 3, introduces the 3-demsional MCvD system model is introduced. Section 4 introduces proposed channel model. Performance evaluation is presented in section 5 and section 6 concludes the paper.

\section{LITERATURE SURVEY}

As shown in literature [13], a new modulation technique based on the method of using different types of molecules for consecutive symbol time slots. This technique referred as Molecular Concentration Shift Keying (MOCSK). The work in [13], also proposes a new system model for propagation of molecules and the propagation channel based on the Poisson Distribution. This model is more realistic and efficient than the channel based on the Binomial Distribution. The paper evaluates and compares the probability of error for Binary and Quaternary Concentration Shift Keying, Molecular Shift Keying (MOSK) and the proposed MOCSK modulation technique. Numerical results show that the proposed MOCSK technique has lower probability of error as compared to CSK and MOSK techniques.

In [14], an energy efficient ISI mitigation technique for MCvD channel is proposed. Three different approaches are considered for energy efficiency problem in the MCvD system. The first approach is to describe energy efficient modulation techniques i.e. Molecular Transition Shift Keying (MTSK), and it is designed to reduce the ISI for a single 
transmitter and a single receiver. When the transmitter sends more than one consecutive symbol ' 1 ', then it is hard for the decoder to detect symbol ' 0 ' after these symbols due to the effect of ISI. For this problem, MTSK is employed in which two types of molecules are used, denoted as type-A and type-B with same number of molecules to encode the symbol ' 1 ' and no molecules for symbol ' 0 '. The type-B molecules are emitted before each symbol ' 0 ' instead of typeA molecules, as a result ISI observed by symbol ' 0 ' decreases.

The second approach reported in [14], introduces a technique to determine the optimal detection threshold values that minimize the bit error rate. If there is a slight change in the system parameters such as temperature, distance, diffusion co-efficient, radius of the receiver etc, then it needs to repeat the calculation of threshold.

The Power Adjustment (PA) is the third approach reported in [14], utilizes the residue molecules from previous symbol and also reduces the energy consumption in the transmitter node. For sending consecutive symbols ' 1 ', the same amount of messenger molecules is emitted for each symbol. This increases the amount of molecules in the channel in the form of residual molecules and cause ISI for the following symbols. Simulation results comparing the error performance of CSK-PA, MoSK-PA, MTSK-PA, the results shows that PA technique reduces the ISI significantly.

In the literature [15], the problem of time synchronization is discussed in concentration based molecular communication via diffusion system. The problem of time synchronization arises when the transmitter and receiver nano-machines have non-synchronous clock resulting in timing offset delay. Due to this delay, receiver may not identify the beginning of the each symbol duration. To overcome this problem, the paper introduces a training based synchronization and Unbiased Linear Estimation technique to efficiently estimate the value of timing offset by the sequence of molecule arrival times measured by the receiver to have synchronization with the transmitter.

In [16], a non-coherent detection scheme has been shown to mitigate ISI in the diffusion based molecular communication system. in this scheme, a low complexity molecular receiver is considered. This scheme is able to achive moderate data rate, low computational complexity and less error rates compared with the coherent Maximum A Posterior (MAP) or Minimum Mean Square Error (MMSE). The coherent scheme requires accurate channel state information (CSI) but the CSI cannot easily estimate because of diffusion dynamics and channel disturbance. The noncoherent detection is based on the difference of received molecular concentration and it is independent from the diffusion channel conditions. The simulation results demonstrate that non-coherent has low computational complexity as it excludes the matrix operations and require no channel information.

In order to mitigate ISI, a Poisson distribution channel model is proposed in this literature. In this channel model, the messenger molecules are propagating using Poisson random variable $\Delta X_{P}$. Compared to both distribution channel models, the proposed channel model is capable of suppressing ISI completely. Simulation results demonstrate that not only the Inter-Symbol Interference (ISI) suppressed, but the Symbol Error Rate is also reduced. Analytical results are verified by 100 times simulations in MUCIN simulator for a 3-dimensional environment.

\section{III.3-D SYSTEM MODEL}

The TN and RN are stationary devices and they are placed in 3-D aqueous environment of uniform viscosity and temperature. The size of the TN has no volume that represent it is a point source and it is placed at Cartesian coordinates $(-d, 0,0)$. The fully absorbing spherical $\mathrm{RN}$ of radius $r_{R N}$ and receiver is located inside the receptor space have spherical shape. The receiver has ability to count the number of absorbed molecules between two time instants. The transmitter is located at distance $r_{o}$ from the center of the receiver and distance $\mathrm{d}$ from the surface of the receiver, hence, $\left(d=r_{o}-r_{R N}\right)$ [5]. The propagating information molecules can hit the spherical RN of radius $r_{r}$. Once a randomly propagating information molecule collides with the surface of the sphere, it is absorbed and removes from the environment as illustrated in figure 1. This absorption process is called the First hitting process [8]. The first hitting probability of the diffusing molecules arriving at a spherical receiver $\Omega_{r}$ is formulated as:

$$
F_{\text {hit }}^{3 D}=\frac{r_{R N}}{r_{o}} \frac{r_{o}-r_{R N}}{\sqrt{4 \pi D t^{3}}} e^{\frac{-\left(r_{o}-r_{R N}\right)^{2}}{4 D t}}
$$

The fraction of molecules absorbed by the receiver until time ' $t$ ' is given by

$$
N_{\text {hit }}^{3 D}\left(\Omega_{r}, \frac{t}{r_{o}}\right)=\frac{r_{r}}{r_{o}} \operatorname{erfc}\left[\frac{r_{o}-r_{R N}}{\sqrt{4 \pi D t}}\right]
$$

The received molecular signal consists of three sources of molecules in symbol duration $t_{s}$ : molecules belonging to the current symbol, molecules belonging to the previous symbol and environmental molecules act as a noise [6]. In 
molecular communication, the time is divided into equal sized time slots in which only one symbol can be send. This time slot is called symbol duration and denoted by $t_{s}$. The randomly propagating molecules have probabilistic behavior due to Brownian motion, molecules have no guarantee to arrive at the receiver; instead they have certain probability to hit the receiver. When ' $n$ ' number of molecules is released from transmitter at the start of symbol duration and it has a certain probability of hitting molecules $P_{\text {hit }}$ at the receiver. Assuming that each molecule are moving independently (i.e., no collisions between the information carrying molecules). This probability of hitting molecules at the receiver depends upon symbol duration $t_{s}$, the distance between transmitter and receiver $d$ and is denoted by $P_{h i t}\left(d, t_{s}\right)$ [9]. If n molecules are released at the start of symbol duration, the number of received molecules within the current symbol duration $\left(N_{c(n)}\right)$ is a random variable and it follows a binomial distribution function that is given as:

$$
N_{c(n)} \sim \operatorname{Binomial}\left(n, P_{h i t}\left(d, t_{s}\right)\right)
$$

The total number of received molecules during a single symbol interval is composed of three parts: (a) The molecules received at the current symbol $N_{c(n)}$, (b) The residue molecules from the previous symbol $N_{P(n)}$, and (c) The molecules from other sources that can be summed up as a noise $N_{n}$. A binomial distribution (Binomial $\left.(n, p)\right)$ can be approximated with a normal distribution function with zero mean and $\sigma^{2}$ variance and it is defined as:

$$
N \sim \operatorname{Normal}\left(0, \sigma^{2}\right)
$$

Due to the Brownian motion, the information carrying molecules have probabilistic behavior. All molecules are not guaranteed to reach at the receiver, rather they have probability of hitting the receiver in a given time interval. $N_{\text {hit }}$ is the number of received molecules in symbol duration and it is composed of number of received molecules from the current symbol duration and the molecules from previous symbol duration [10].

$$
N_{\text {hit }}=N_{P(n)}+N_{C(n)}
$$

The current received symbol $N_{C(n)}$ is depending upon the current transmitted symbol and the previous intended symbol. The noise in the communication system is Additive White Gaussian Noise (AWGN) and the noise is also a random variable that follows a normal distribution function with zero mean and $\sigma^{2}$ variance [11]. In this literature, we compare Poisson and Gaussian approximations considering without and with the effect of noise in the MCvD system.

\section{IV.POISSON DISTRIBUTION CHANNEL MODEL}

The messenger molecules are the information carrying particles in molecular scale. These molecules are released from TN and spread out in the fluid medium by diffusion. Diffusion is the random movement of information particles from an area of higher concentration in the area of lower concentration. The random movement of particles is modeled by Brownian motion. The motion is governed by the combined forces put on the information carrying particles by the molecules of liquid, which are in regular motion due to thermal energy present in the medium. Therefore, no source of energy is used for diffusion processes [12].

In traditional molecular communication, diffusion of single information molecules in unit time is a random variable $(\Delta X)$, which follows Gaussian distribution (also called Normal Distribution) function as shown in figure 1. This is a continuous probability distribution that has zero mean. The displacement of the information molecules has variance of $(2 \mathrm{D} \Delta \mathrm{t})$ in a $\Delta \mathrm{t}$ length of time and we have:

$$
\Delta \mathrm{X} \sim \operatorname{Normal}(\text { mean, variance) }
$$

$$
\Delta \mathrm{X} \sim \mathrm{N}(0,2 \mathrm{D} \Delta \mathrm{t})
$$

where $\mathrm{D}$ is the diffusion co-efficient and $\Delta \mathrm{t}$ is the step time [9]. Similarly to Gaussian, a Poisson approximation of binomial distribution is proposed in this literature. The proposed Poisson distribution is a discrete probability distribution function. In a 3-D channel, now the displacement of a single information molecule follows Poisson distribution that has equal mean and variance is defined as:

$$
\begin{gathered}
\Delta \mathrm{X}_{\mathrm{P}} \sim \text { Poisson(mean, variance) } \\
\text { mean }=\text { variance }=2 \mathrm{D} \Delta \mathrm{t}
\end{gathered}
$$

The messenger molecules diffuse through a 3-D environment where the movements in all three dimensions are independent distributed Poisson random variable $\Delta \mathrm{X}_{\mathrm{P}}$. We investigate which model is better for representing the channel response of MCvD system. 


\section{RESULTS AND DISCUSSION}

We consider a time slotted MCvD system as each symbol is transmitted in equal slot duration $\left(t_{s}\right)$. In this paper, we consider binary ON-OFF keying modulation in which the transmitter emits ' $\mathrm{N}$ ' number of messenger molecules (MMs) when the symbol to be transmitted is ' 1 '; otherwise the transmitter remains inactive when transmitting symbol ' 0 ' [6]. The received signal is decoded based on the molecular concentration received during a time slot. For numerical analysis, we have assumed the distance between transmitter and $\mathrm{RN}$ and symbol duration are $\mathrm{d}=1 \mu \mathrm{m}$ and $\mathrm{t}_{\mathrm{s}}=$ $0.064 \mathrm{sec}$ respectively and hence they have equal probabilities $\left(\mathrm{P}_{1}=\mathrm{P}_{2}=0.5\right)$ of hitting molecules at the receiver. Table 1 shows simulation parameters used in the system.

\section{TABLE I SIMULATION PARAMETERS}

\begin{tabular}{|l|l|l|}
\hline S.No & Parameters & Values \\
\hline 1 & Number of MM $(\mathrm{s})$ & 1000 \\
\hline 2 & Simulation Step Time $(\Delta \mathrm{t})$ & $1 \mathrm{msec}$ \\
\hline 3 & Sampling Time $\left(\mathrm{t}_{\mathrm{ss}}\right)$ & $5 \mathrm{msec}$ \\
\hline 4 & Number of Symbols & 1 \\
\hline 5 & Symbol Duration $\left(\mathrm{t}_{\mathrm{s}}\right)$ & $64 \mathrm{msec}$ \\
\hline 6 & Diffusion Co-efficient $(\mathrm{D})$ & $79.4(\mu \mathrm{m})^{2} / \mathrm{sec}$ \\
\hline 7 & Replications & 100 \\
\hline 8 & Signal to Noise Ratio $(\mathrm{db})$ & $5 \mathrm{db}$ \\
\hline 9 & Radius of transmitter nano-machine $\left(\mathrm{r}_{\mathrm{TN}}\right)$ & $10 \mu \mathrm{m}$ \\
\hline 10 & Radius of receiver nano-machine $\left(\mathrm{r}_{\mathrm{RN}}\right)$ & $10 \mu \mathrm{m}$ \\
\hline
\end{tabular}

The diffusion co-efficient, $\mathrm{D}=79.4 \mu \mathrm{m}^{2} \mathrm{~s}^{-1}$ is used given that it is known value for insulin in water at human body temperature. We utilize MolecUlar CommunicatIoN (MUCIN) simulator to verify the traditional and proposed channel models in 3-D environment.

\section{A. Hitting Time Analysis}

The impulse response of the molecular communication channel based on the Gaussian distribution model experienced temporal spreading of molecular signal. Such temporal spreading of molecular signal highly affects the performance of the system. It is desirable to have the molecular signal to be spike like and low spreading of the pulse, which reduces the ISI [5].

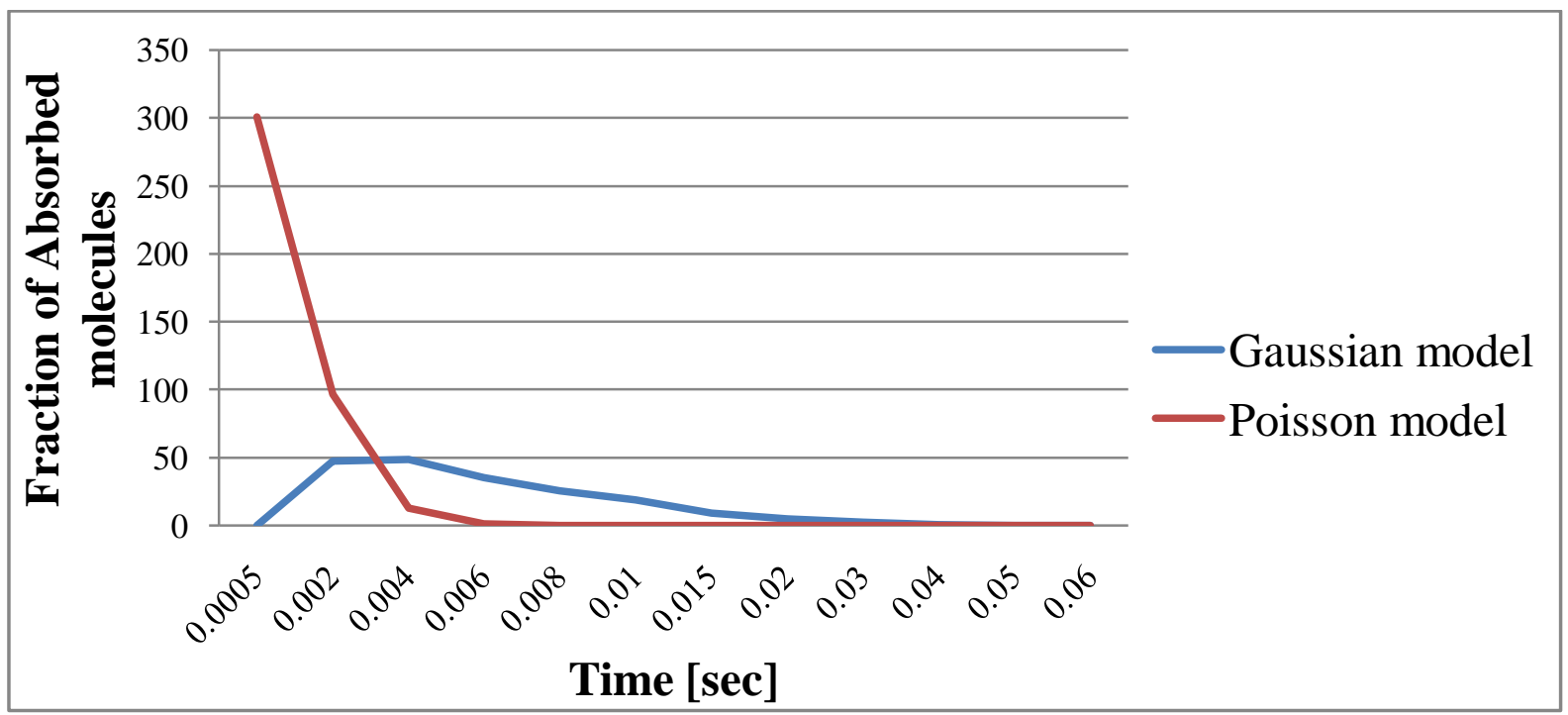

Fig. 2 A fraction of information molecules absorbed by the receiver nano-machine during the first symbol duration

In figure 2, shows the absorption rate of molecules, i.e., the time at which amount of molecules absorb by the receiver. The absorption of information molecules in Poisson distribution is significantly higher at a time $0.0005 \mathrm{sec}$ than that of the conventional Gaussian distribution. Interestingly, the absorption of the molecules decreasing sharply and becomes zero at a time $0.006 \mathrm{sec}$ that reduces spreading of molecular signal. 
B. Improvement on Inter-Symbol Interference

We adopt a simple decoding method at the receiver side: during each symbol duration, the receiver counts total number of information molecules it received, and sees if the number is greater than or equal to threshold value. If yes, symbol 1 is decoded; otherwise, symbol 0 is decoded [6].

When there is continues transmission of symbols, the received molecular concentration is affected by the current and previous symbol emissions. Figure 3(a,b) shows the received molecular concentration measured by RN for each binary symbols in both channel models. Figure 3(a) shows Gaussian distribution channel model that depicts the late arriving molecules (i.e., stray molecules) from previous symbol ' 1 ' often interfere with the symbol ' 0 ', creating ISI. Figure 3(b) shows Poisson distribution channel model that depicts the molecules of previous symbols are faded completely which results in reduced ISI. The decision thresholds for current received symbol are independent of the previous decoded symbol.

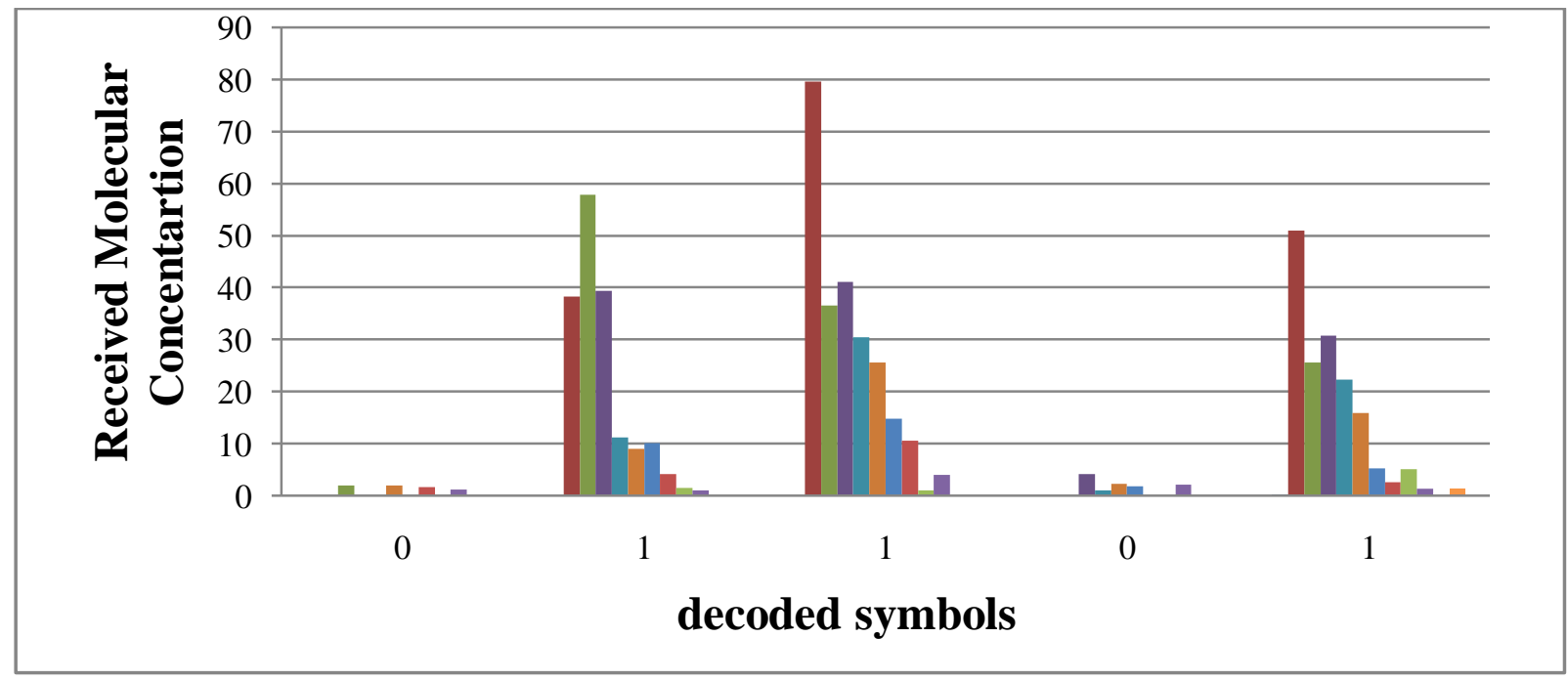

(a)

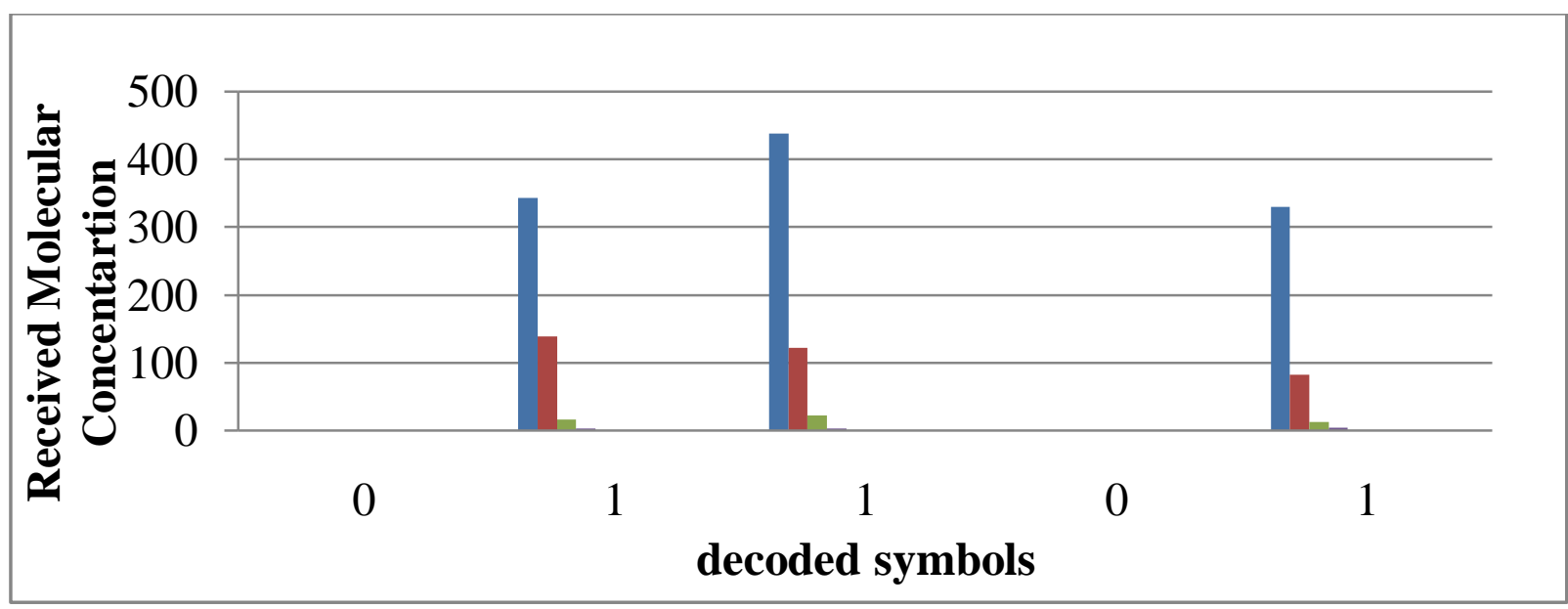

(b)

Fig. 3 The received molecular concentration measured by receiver nano-machine for each binary symbols (01101) (a) for Gaussian Distribution channel model (b) for Poisson Distribution channel model.

C. Symbol Error Rate Analysis

The noise in molecular communication is additive white noise, which is defined as another source release same type of molecules that are used to encode the information. Therefore, the receiver may pick up background noise during each symbol duration, and sense an incorrect molecular concentration level would results in wrong decoding of received symbol, thus producing Symbol Error Rate (SER).

Figure 4 shows the SER comparison using with and without additive noise. It is clear that the SER in both cases are lower when the Poisson distribution model is used. 


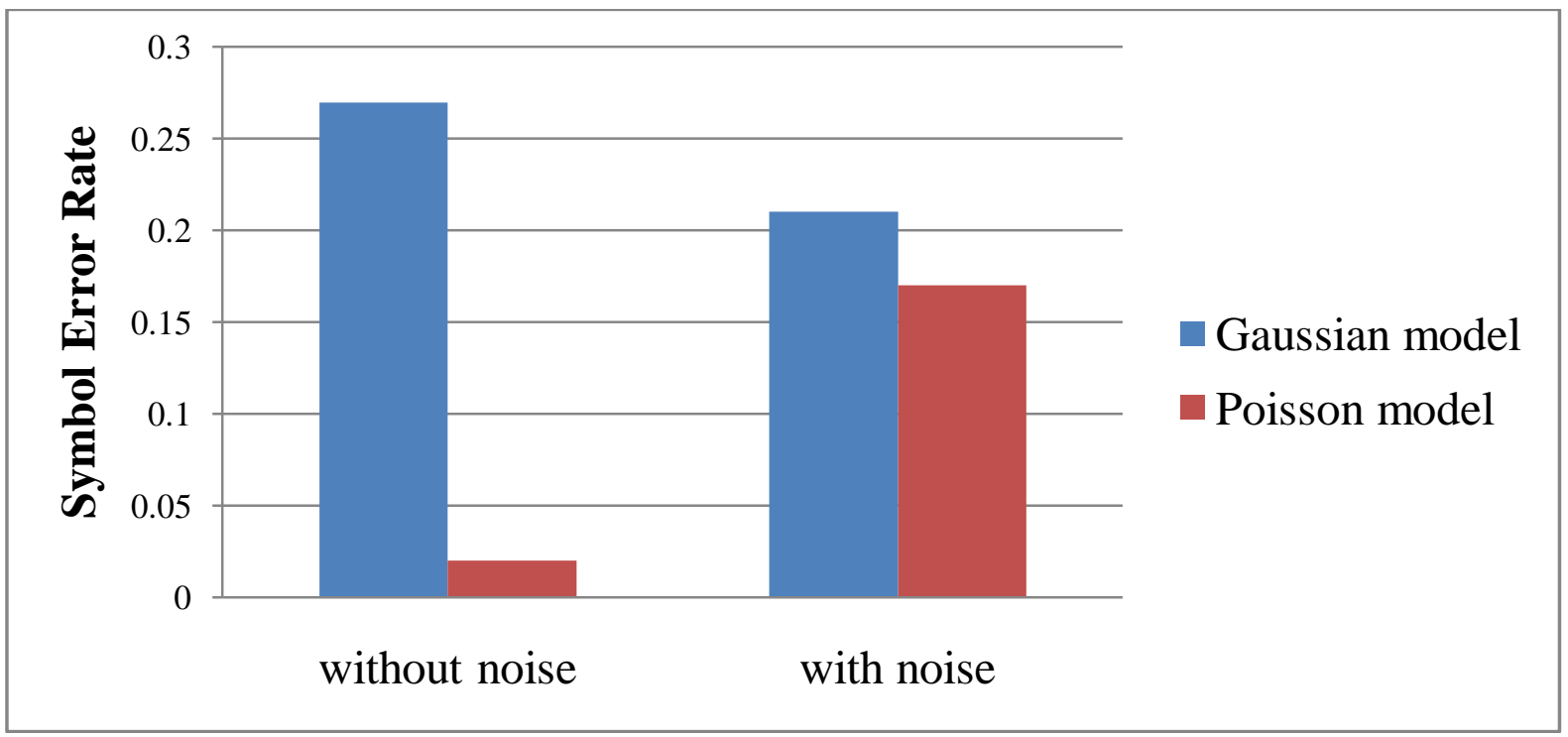

Fig. 4 SER comparison among the Gaussian and the Poisson Distribution channel models.

D. Communication Delay

In $\mathrm{MCvD}$ channel, since the information molecules are propagating randomly, the communication delay of the system is large. Figure 5 shows that the communication delay of molecular signal in the Gaussian distribution model is comparatively less than the communication delay of molecular signal in the Poisson distribution model.

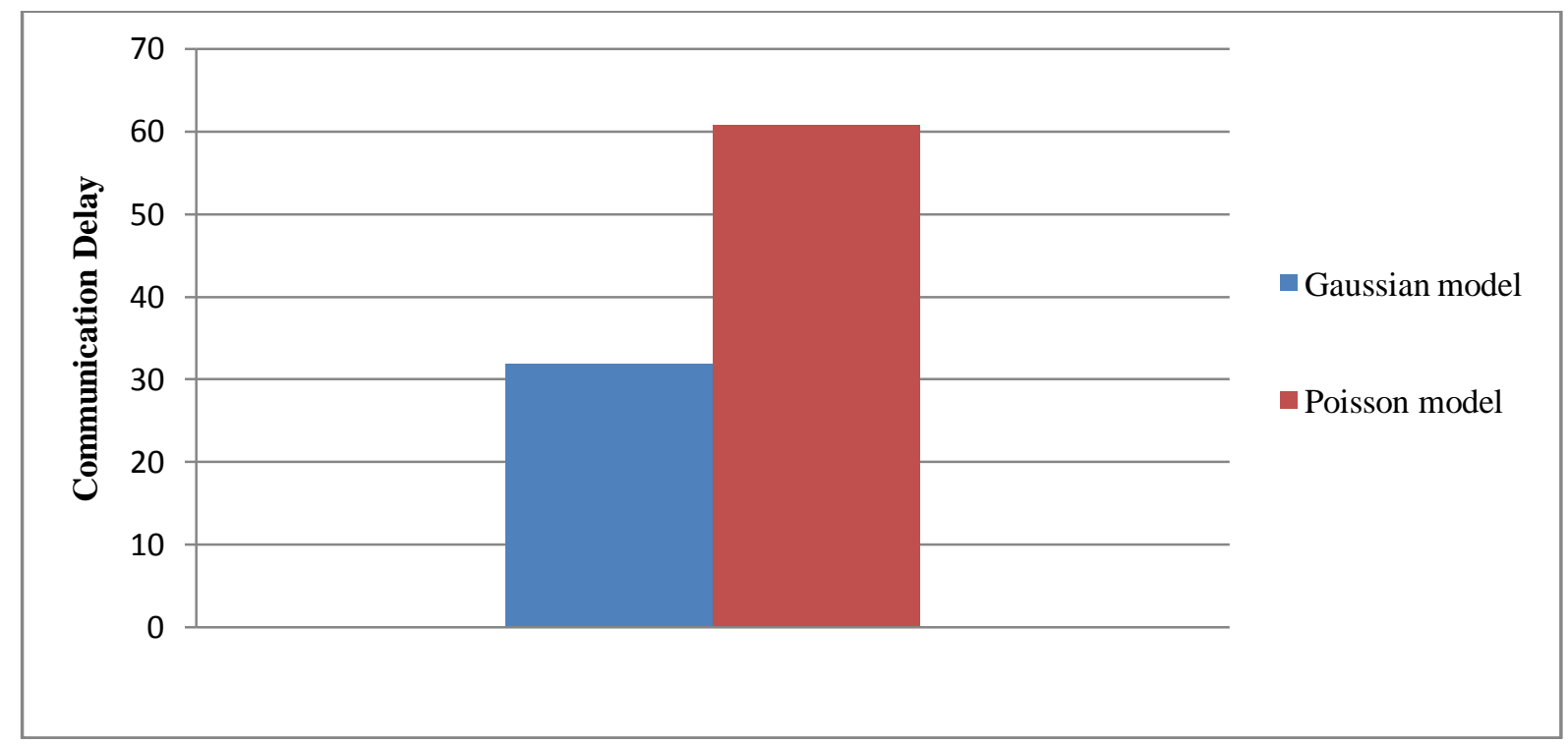

Fig. 5 Communication Delay comparison among the Gaussian and the Poisson Distribution channel models.

\section{VI.CONCLUSION}

In this paper, we have examined the idea of channel model for the diffusion based molecular communication. It has been shown that for diffusion based channel, the traditional Gaussian Distribution channel model is no longer suitable for mitigating ISI. We have investigated a new Poisson Distribution channel model that significantly combat the ISI. This proposed model reduces the additive noise effect present in the diffusion channel and also the previous symbol interference has disappeared. This channel model, however, has the highest communication delay than Gaussian distribution channel model.

\section{REFERENCES}

1. Mahfuz MU, Makrakis D, Mouftah HT, On the Detection of Binary Concentration-encoded Unicast Molecular Communication in Nanonetworks, In BIOSIGNALS, 2011 Jan 26 (pp. 446-449). 
2. Mahfuz MU, Makrakis D, Mouftah HT, On the characterization of binary concentration-encoded molecular communication in nanonetworks, Nano Communication Networks, 2010 Dec 31, 1(4):289-300.

3. Yilmaz HB, Chae CB, Simulation study of molecular communication systems with an absorbing receiver, Modulation and ISI mitigation techniques, Simulation Modelling Practice and Theory, 2014 Dec 31, pp.49:136-50.

4. Mahfuz MU, Makrakis D, Mouftah HT, Strength-based optimum signal detection in concentration-encoded pulse-transmitted OOK molecular communication with stochastic ligand-receptor binding, Simulation Modelling Practice and Theory, 2014 Mar 31, 42:pp.189-209.

5. Yilmaz HB, Chae CB, Tepekule B, Pusane AE, Arrival modeling and error analysis for molecular communication via diffusion with drift, in Proc: on the Second Annual International Conference on Nanoscale Computing and Communication, 2015 Sep 21, pp. 26, ACM.

6. Ko PY, Lee YC, Yeh PC, Lee CH, Chen KC, A new paradigm for channel coding in diffusion-based molecular communications, Molecular coding distance function, in: Global Communications Conference (GLOBECOM), IEEE 2012 Dec 3 (pp. 3748-3753).

7. ShahMohammadian, Hoda, System design for nano-network communications, PhD. Dissertation, University of Calgary, 2013.

8. Heren AC, Yilmaz HB, Chae CB, Tugcu T, Effect of degradation in molecular communication: Impairment or enhancement?, IEEE Transactions on Molecular, Biological and Multi-Scale Communications, 2015 June1(2), pp.217-29.

9. Farsad, Nariman, Gerald Audette, and Uyen Trang Nguyen, Molecular Communication, 2014.

10. Kuran MŞ, Yilmaz HB, Tugcu T, Özerman B, Energy model for communication via diffusion in nanonetworks, Nano Communication Networks, 2010 Jun 30, 1(2), pp.86-95.

11. Kuran MS, Yilmaz HB, Tugcu T, Akyildiz IF, Modulation techniques for communication via diffusion in nanonetworks, in: IEEE in ternational conference on communications (ICC), 2011 Jun 5, pp. 1-5.

12. Yilmaz HB, Heren AC, Tugcu T, Chae CB, Three-dimensional channel characteristics for molecular communications with an absorbing receiver, IEEE Communications Letters, 2014 Jun, 18(6), pp.929-32.

13. Arjmandi H, Gohari A, Kenari MN, Bateni F., Diffusion-based nanonetworking: A new modulation technique and performance analysis, IEEE Communications Letters. 2013 Apr, 17(4), pp.645-8.

14. Tepekule B, Pusane AE, Yilmaz HB, Chae CB, Tugcu T., ISI mitigation techniques in molecular communication, IEEE Transactions on Molecular, Biological and Multi-Scale Communications, 2015 Jun, 1(2), pp.202-16.

15. Hsu BK, Lee CH, Yeh PC, On Timing Synchronization for Quantity-based Modulation in Additive Inverse Gaussian Channel with Drift, arXiv preprint arXiv:1411.2443, 2014 Nov 10.

16. Li B, Sun M, Wang S, Guo W, Zhao C., Low-complexity noncoherent signal detection for nanoscale molecular communications, IEEE transactions on nanobioscience, 2016 Jan, 15(1), pp.3-10.

\section{BIOGRAPHIES}

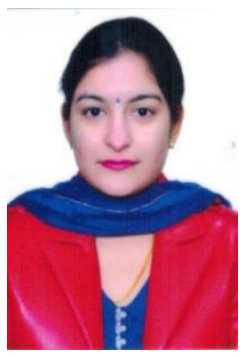

Anmol Preet Kaur was born in Amritsar, Punjab, India in 1992. She received the Bachelor's degree in the Electronics and Communication Engineering, from Amritsar College of Engineering and Technology, Amritsar, Punjab, India in 2014. Presently, she is pursuing her Master's under the guidance of Dr. Deep Kamal Kaur Randhawa at Guru Nanak Dev University Regional Campus Jalandhar, Punjab, India. Her research interest focuses on Mobile Adhoc Networks and Molecular Communication.

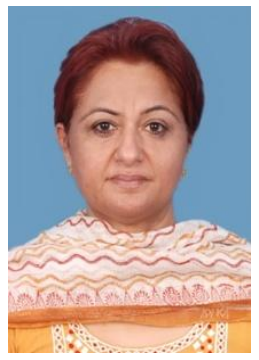

Deep Kamal Kaur Randhawa was born in Ropar, Punjab, India in 1970. She received the Bachelor's degree in Electronics and Electrical Communication Engineering from Guru Nanak Dev Engineering College, Ludhiana, Punjab, India in 1991.Then she did her Master's from Guru Nanak Dev Engineering College, Ludhiana, Punjab, India in 2003 and Doctorate from Guru Nanak Dev University, Amritsar in 2012.She is presently serving as a Senior Assistant Professor in the Department of Electronics and Communication Engineering, Guru Nanak Dev University, Regional Campus Jalandhar, Punjab, India. Her professional research interest is focused on study and analysis of nanomaterials for potential applications in nanoelectronics. She is Fellow Member IETE, Member The Institution of Engineers (India) and Life Member ISTE, New Delhi. She was awarded the Best Teacher of Punjab in 2007 by ISTE, New Delhi. 